\title{
Indicadores antropométricos, mas não a aptidão aeróbia, se associam com a reatividade vascular de pressão arterial em homens
}

\author{
Anthropometric parameters, but not aerobic fitness, associates with \\ vascular reactivity of blood pressure in men
}
Reginaldo Luiz Nascimento, ${ }^{1}$ Francisco Navarro, ${ }^{2}$ Mário Sevilio Junior, ${ }^{2}$ Ricardo Ariel Costa Souza, ${ }^{1}$ Sérgio Rodrigues Moreira ${ }^{1 *}$

ARTIGO ORIGINAL | ORIGINAL ARTICLE

\begin{abstract}
O objetivo deste estudo foi verificar a associação entre indicadores antropométricos e aptidão aeróbia com a reatividade vascular de pressão arterial $\left(\mathrm{RV}_{\mathrm{PA}}\right)$ em homens saudáveis. Quarenta indivíduos do sexo masculino $(27.3 \pm 6.3$ anos; $77.2 \pm 11.5 \mathrm{~kg} ; 175.7 \pm 6.9 \mathrm{~cm})$ realizaram medidas de índice de massa corporal (IMC), circunferência da cintura (CC), relação cintura/quadril (RCQ), percentual de gordura (\%G), consumo máximo de oxigénio $\left(\mathrm{VO}_{2} \mathrm{max}\right.$ ) e um teste de $\mathrm{RV}_{\mathrm{PA}}$ (Cold Pressor Test - CPT) com imersão da mão em água a $4^{\circ}$ Celsius durante 1 minuto. Não ocorreu associação entre aptidão aeróbia $\left(\mathrm{VO}_{2}\right.$ max abaixo ou acima da média e teste de correlação) com $\operatorname{RV}_{\mathrm{PA}}(p>0.05)$. Indicadores antropométricos como IMC $(r=0.31 / 0.54)$, CC $(r=0.30 / 0.49)$, RCQ $(r=0.30 / 0.36)$ e \%G $(r=$ 0.31 / 0.35) demonstraram correlações significativas com $\mathrm{RV}_{\mathrm{PA}}(p<0.05)$, contudo, somente a CC durante o CPT $(\beta=0.30 / 0.43)$ e o IMC após o CPT $(\beta=0.54 / 0.81)$ foram preditores da $\operatorname{RV}_{\mathrm{PA}}(p \leq$ 0.05). Conclui-se que indicadores antropométricos apresentaram correlações independentes com a $\mathrm{RV}_{\mathrm{PA}}$, contudo, apenas a CC (durante) e o IMC (após) se mostraram preditores significativos da $\mathrm{RV}_{\mathrm{PA}}$, o que sugere que a adiposidade corporal, independente da aptidão aeróbia, tenha importante papel como fator de risco para a doença cardiovascular.

Palavras-chave: adiposidade corporal, antropometria, aptidão física, reatividade vascular
\end{abstract}

ABSTRACT

The aim of this study was to verify the association between anthropometric parameters and aerobic fitness with vascular reactivity of blood pressure $\left(\mathrm{VR}_{\mathrm{BP}}\right)$ at healthy male subjects. It was assessed in forty apparently healthy male $(27.3 \pm 6.3$ years; $77.2 \pm 11.5 \mathrm{~kg} ; 175.7 \pm 6.9 \mathrm{~cm})$ the body mass index (BMI), waist circumference (WC), waist-rip ratio (WRR), body fat (\%BF), maximum oxygen uptake $\left(\mathrm{VO}_{2} \mathrm{max}\right)$ and a VR test of BP (Cold Pressor Test - CPT) with immersion of hand in the water $\left(4^{\circ}\right.$ Celsius during $1 \mathrm{~min})$. There was no association between aerobic fitness $\left(\mathrm{VO}_{2} \mathrm{max}\right.$ below or above of mean and relationship test) and $\mathrm{VR}_{\mathrm{BP}}(p>0.05)$. Anthropometric indicators as BMI $(r=0.31 / 0.54)$, WC $(r=0.30 / 0.49)$, WRR $(r=0.30 / 0.36)$ and $\% \mathrm{BF}(r=0.31 / 0.35)$ showed positive relationships with $\mathrm{VR}_{\mathrm{BP}}(p<0.05)$. However, only WC during the CPT $(\beta=0.30 / 0.43)$ and BMI after the CPT $(\beta=$ $0.54 / 0.81)$ were predictors of $\mathrm{VR}_{\mathrm{BP}}(p \leq 0.05)$. We conclude that anthropometric indicators showed independents relationships with $\mathrm{VR}_{\mathrm{BP}}$. Nevertheless, only the WC (during) and BMI (after) were significant predictors of $\mathrm{VR}_{\mathrm{BP}}$, suggesting that body adiposity, independent of aerobic fitness, have an important role as a cardiovascular risk factor.

Keywords: body adiposity, anthropometric, physical fitness, vascular reactivity

Artigo recebido a 02.05.2013; $1^{\text {a }}$ Revisão 09.07.2013; 2 ${ }^{\mathrm{a}}$ Revisão 03.11.2013; Aceite 13.01.2014

${ }^{1}$ Universidade Federal do Vale do São Francisco (UNIVASF), Petrolina, PE - Brasil

${ }^{2}$ Universidade Federal do Maranhão (UFMA), São Luís - MA, Brasil

* Autor correspondente: Universidade Federal do Vale do São Francisco, CEFIS - Educação Física, Av. José de Sá Maniçoba, S/N, Centro, CEP: 56304205 Petrolina - PE, Brasil; E-mail: serginhocapo@gmail.com 


\section{INTRODUÇÃO}

A prática de exercícios físicos tem sido amplamente destacada nas diferentes populações, com fim de melhora na aptidão física do praticante (Gaesser, Angadi, \& Sawyer, 2011). Por outro lado, uma baixa aptidão física poderá estar inversamente correlacionada com a gordura corporal (Palou, Serra, Bonet, \& Picó, 2000) a qual em excesso torna-se um importante fator de risco para doenças cardiovasculares, entre as quais se destaca a hipertensão arterial sistémica (HAS) (Chobanian et al., 2003; Triches \& Giugliani, 2005).

Classicamente, a HAS tem sido associada com uma aumentada reatividade vascular de pressão arterial $\left(\mathrm{RV}_{\mathrm{PA}}\right)$ durante e após momentos agudos de estresse induzido (Hines \& Brown, 1936; Wood, Sheps, Elveback \& Schirger, 1984). A elevação significativa da $R V_{P A}$ pode sugerir uma prejudicada modulação autonómica e tónus simpático aumentado, fenómeno associado ao risco cardiovascular em médio e longo prazo (Brownley et al., 2003; Flaa, Mundal, Eide, Kjelsen \& Rostrup, 2006; Galetta et al., 2006; Su et al., 2006) e que apresenta relação positiva com a adiposidade corporal (Kuniyoshi et al., 2003; Ribeiro et al., 2001).

Segundo Christou et al. (2005), indicadores de adiposidade corporal e a potência aeróbia estão associados a fatores de risco metabólicos e hemodinâmicos. Um reduzido consumo máximo de oxigénio pode minimizar o efeito cardioprotetor da aptidão aeróbia num indivíduo (Thompson et al., 2003). Se associado a isso, ainda um aumentado conteúdo de gordura corporal estiver instalado, o risco cardiovascular aumenta (Vicent et al., 2011; Wilson, D'Agostino, Sullivan, Parise, \& Kannel, 2002). Entretanto, a baixa aptidão aeróbia e ainda a aumentada adiposidade corporal, em geral, se combinam (Hoehner et al., 2008), o que traz dúvidas quanto aos efeitos diretos e independentes de cada uma dessas condições, bem como, do poder de predição dessas variáveis, na associação com fatores de risco cardiovasculares. Deste modo, ainda são necessários estu- dos que quantifiquem o grau de associação entre aptidão aeróbia e indicadores antropométricos de adiposidade corporal com fatores de risco cardiovascular. Além disso, os estudos supracitados não apresentam o poder de predição destes indicadores nos fatores de risco hemodinâmicos, o que poderia ter importância clínica na área da avaliação da saúde cardiovascular. Sendo assim, os objetivos do presente estudo foram: 1) Verificar a associação independente entre indicadores antropométricos e aptidão aeróbia com a $\mathrm{RV}_{\mathrm{PA}}$; e, 2) Analisar o poder de predição das variáveis associadas com a $\mathrm{RV}_{\mathrm{PA}}$ em homens adultos. A hipótese do presente estudo é que a potência aeróbia não apresentará correlação direta com $\mathrm{RV}_{\mathrm{PA}}$. Por outro lado, indicadores antropométricos de fácil aplicação apresentarão correlação direta e significativo poder de predição da $R V_{\mathrm{PA}}$.

\section{Participantes}

\section{MÉTODO}

Cálculo amostral prévio foi realizado a partir do software GPower 3.1. Ao considerar um Effect Size $=0.21 ; \alpha=5 \%$; e Power $=0.80$, o tamanho da amostra requerido para os objetivos propostos e quantidade de variáveis analisadas foi de 40 participantes.

A partir disso, 40 indivíduos do sexo masculino, aparentemente saudáveis com idade entre 17-46 anos foram convidados a participar do estudo, o qual se caracterizou como do tipo transversal e com enfoque biológico. Dos 40 voluntários foi possível realizar todas as mensurações propostas em 30 participantes. Dez participantes não realizaram as medidas de aptidão aeróbia, porém, foram mantidos na amostra para análises posteriores. A tabela 1 apresenta as características gerais da amostra estudada. O Comitê de Ética em Pesquisa da Universidade local aprovou a pesquisa (proc. 5934.0.000.441-10) e todos os participantes foram informados dos procedimentos a serem adotados e assinaram um Termo de Consentimento Livre e Esclarecido em acordo a resolução 196/96 do Conselho Nacional de Saúde em Pesquisa com Seres Humanos. 


\section{Instrumentos e Procedimentos}

A análise da composição corporal através de parâmetros antropométricos e a aptidão aeróbia através da medida indireta do consumo máximo de oxigénio foram realizadas para posterior verificação da associação desses parâmetros com a $\mathrm{RV}_{\mathrm{PA}}$ mensurada pelo cold Pressor Test.

\section{Indicadores Antropométricos}

O peso corporal foi verificado através de uma balança de plataforma digital da marca Tech Lyne (Petrolina/PE/Brasil) com precisão de $0.1 \mathrm{~kg}$.

Para estatura utilizou-se um estadiómetro de metal da marca Physical (Londrina, PR Brasil) com precisão de $0.1 \mathrm{~cm}$. O índice de massa corporal (IMC) foi calculado através da relação peso/estatura ${ }^{2}$.

Dobras cutâneas das regiões tricipital, supra-ilíaca e abdominal, foram mensuradas de forma rotacional e replicadas três vezes no hemicorpo direito do avaliado. $\mathrm{O}$ valor médio foi considerado para estimativa da densidade corporal em acordo a equação preditiva de Jackson e Pollock (1978). O percentual de gordura (\%Gordura) foi calculado de acordo com a fórmula de Siri $(1961)=(4.91 /$ densidade $4.5) \times 100$ (Guedes \& Guedes, 1997). O equipamento utilizado foi um adipómetro da marca Cescorf (Porto Alegre, RS - Brasil) com precisão de $0.1 \mathrm{~mm}$. Circunferências da cintura e quadril foram analisadas utilizando uma fita métrica metálica inextensível com precisão de $0.1 \mathrm{~cm}$ da marca Cescorf (Porto Alegre, RS Brasil) conforme as técnicas descritas por Callaway et al. (1988). A relação cintura/quadril (RCQ) foi calculada e todas as medidas foram realizadas por um único avaliador.

\section{Aptidão Aeróbia}

A aptidão aeróbia foi avaliada a partir do consumo máximo de oxigénio $\left(\mathrm{VO}_{2} \mathrm{max}\right)$ indireto. Teste de corrida para desempenho máximo em 1600 metros $(1600 \mathrm{~m})$ foi realizado, onde o avaliado foi orientado a realizar a distância alvo no seu menor tempo possível. Atra- vés da velocidade média (distância/tempo) no teste de $1600 \mathrm{~m}$ (VM1600m) calculou-se o $\mathrm{VO}_{2} \max$ pela equação: $\mathrm{VO}_{2} \max =0.177 \times$ VM1600m + 8.101. A equação utilizada foi validada por Almeida et al. (2010).

\section{Mensuração da Pressão Arterial (PA) de Repouso}

A mensuração da PA foi realizada por um avaliador experiente e adotou-se o método auscultatório, de acordo com Perloff et al. (1993). Utilizou-se um estetoscópio (Duo Sonic/BD, Juiz de Fora, MG - Brasil) e um esfigmomanómetro da marca Missouri ${ }^{\circledR}$ devidamente calibrado e certificado pelo INMETRO (Missouri, São Paulo, SP - Brasil). As medidas foram realizadas no braço esquerdo com o indivíduo sentado em local calmo e confortável durante um período de 20 minutos de repouso, com mensurações a cada 5 minutos para se considerar a média como representativa do repouso.

O pesquisador responsável pelas mensurações de PA foi submetido a um procedimento de validação das medidas auscultatórias adotadas. Para tal utilizou-se um aparelho automático clinicamente validado por Stergiou, Tzamouranis, Protogerou, Nasothimiou e Kapralos (2008) da marca Microlife modelo BP3AC1-1 (Microlife/Co, USA). O procedimento de validação envolveu 30 voluntários de ambos os sexos, os quais permaneceram por um período aproximado de 10 minutos em repouso e logo após, em ordem randomizada, passaram por avaliações de PA Sistólica (PAS) e PA Diastólica (PAD) pelo método automático e auscultatório. As correlações encontradas para as medidas de PAS e PAD entre métodos automático e auscultatório foram $r=0.90(p<0.001)$ e $r=0.80(p<0.001)$, respetivamente. Além disso, a técnica de Bland e Altman (1986) demonstrou concordância entre as medidas realizadas pelo método automático e auscultatório. A média das diferenças, com $95 \%$ de intervalo de confiança, foi de $3.9(-12.4 / 20.2)$ e $-3.4(-17.3 / 10.5)$ para a PAS e a PAD, respetivamente. 
Mensuração da Reatividade Vascular de Pressão Arterial $\left(R V_{P A}\right)$

A mensuração da $R V_{P A}$ foi realizada através do Cold Pressor Test (CPT) seguindo procedimentos de validação descritos por Hines e Brow (1936). A especificidade do CPT foi comprovada por Bring e Oerting (1933). O protocolo do teste de $\mathrm{RV}_{\mathrm{PA}}$ foi realizado com a mão direita (até altura do punho) do voluntário sendo imersa em recipiente com água em temperatura de $4^{\circ} \mathrm{C}$ durante 1 minuto. Durante os testes de $\mathrm{RV}_{\mathrm{PA}}$ a água foi controlada por termómetro (Incoterm ${ }^{\circledR}$, Porto Alegre, RS). Medidas de PA foram realizadas aos 30s e 60s do teste através do método auscultatório (supracitado). Os valores de pico encontrados durante o teste de $\mathrm{RV}_{\mathrm{PA}}$ foram considerados como Pico30" e Pico60" de PA (Hines \& Brow, 1936; Wood et al., 1984). Aos 2 minutos da recuperação do CPT considerou-se o momento Rpós2'.

\section{Análise Estatística}

A estatística descritiva foi adotada. A normalidade dos dados foi testada pelo teste Shapiro-Wilk. A correlação linear de Pearson foi aplicada para verificar correlações independentes. O método de Bland e Altman (1986) foi utilizado para verificar o nível de concordância entre medidas de PA por método automático e auscultatório. O teste $t$ de Student para amostras independentes foi utilizado para comparar grupos. A regressão múltipla foi realizada entre as variáveis dependentes da $R V_{P A}$ e as variáveis independentes dos indicadores antropométricos. Segundo Hair, Black, Babin, Anderson e Tatham (2009), na regressão múltipla para cada variável independente devem existir no mínimo cinco casos. No presente estudo foi possível realizar a técnica de regressão entre cada variável dependente com as quatro variáveis independentes com um número de 40 indivíduos, ficando uma razão de 10:1 (casos/variável independente).

O nível de significância do estudo foi $p<$ 0.05 e o software foi o SPSS 15.0.

\section{RESULTADOS}

A tabela 1 apresenta as características antropométricas, aptidão aeróbia, PA de repouso e $\mathrm{RV}_{\mathrm{PA}}$ na amostra investigada. Destaca-se a significância estatística do CPT em induzir a $\mathrm{RV}_{\mathrm{PA}}$ para PAS e PAD na amostra investigada $(p<0.01)$.

A tabela 2 apresenta as correlações entre os indicadores antropométricos e a aptidão aeróbia com as variáveis de PA de repouso e $\mathrm{RV}_{\mathrm{PA}}$. $\mathrm{A}$ aptidão aeróbia representada pelo $\mathrm{VO}_{2} \max$ não apresentou correlação direta com $\mathrm{RV}_{\mathrm{PA}}$ $(p>0.05)$. Por outro lado, indicadores antropométricos, especialmente a circunferência da cintura e o IMC apresentaram correlações significativas com a $\mathrm{RV}_{\mathrm{PA}}$ tanto durante como após o CPT $(p<0.05)$.

Com objetivo de identificar uma possível associação entre o $\mathrm{VO}_{2}$ max e a $\mathrm{RV}_{\mathrm{PA}}$, agora em função da comparação de valores médios encontrados na amostra, a tabela 3 apresenta uma subdivisão de indivíduos com $\mathrm{O} \mathrm{VO}_{2} \max$ abaixo e acima da média encontrada. Foi possível identificar que na amostra investigada não existe associação do $\mathrm{VO}_{2}$ max com a PA de repouso, $\mathrm{RV}_{\mathrm{PA}}$ durante e após o CPT $(p>0.05)$.

Apesar de não ser objetivo principal do presente estudo, a tabela 4 demonstra que existe uma associação entre $\mathrm{VO}_{2} \max$ e os indicadores antropométricos, representada pelas variáveis IMC, circunferência da cintura e $\%$ de gordura $(p<0.05)$.

A técnica de regressão múltipla aplicada entre os indicadores antropométricos e as variáveis da $\mathrm{RV}_{\mathrm{PA}}$, demonstrou que a $\mathrm{CC}$ é uma preditora significativa da $\mathrm{RV}_{\mathrm{PA}}$ durante o CPT. Já na recuperação do estresse induzido, evidenciou-se que o IMC pode ser considerado um bom preditor $(p<0.05)$.

\section{DISCUSSÃO}

Os principais achados do presente estudo evidenciaram correlações independentes de indicadores antropométricos, principalmente da CC e do IMC, com a $\mathrm{RV}_{\mathrm{PA}}$ durante e após o estresse induzido (Tabela 2). Ainda, foi 
16 | RL Nascimento, F Navarro, M Sevilio Junior, RAC Souza, SR Moreira

Tabela 1

Média $( \pm D P)$ das características antropométricas, aptidão aeróbia, pressão arterial de repouso e reatividade vascular

\begin{tabular}{|c|c|c|c|c|}
\hline & $\begin{array}{l}M \pm D P \\
(n=30)\end{array}$ & Variação & $\begin{array}{l}M \pm D P \\
(n=10)\end{array}$ & Variação \\
\hline Idade (anos) & $25.7 \pm 5.5$ & $17.0 / 39.0$ & $32.0 \pm 6.3$ & $24.0 / 46.0$ \\
\hline Peso (kg) & $75.4 \pm 11.3$ & $55.4 / 105.0$ & $82.7 \pm 11.1$ & $63.0 / 95.5$ \\
\hline Estatura $(\mathrm{cm})$ & $174.5 \pm 6.2$ & $164.0 / 188.0$ & $179.2 \pm 8.2$ & $168.0 / 192.0$ \\
\hline $\operatorname{IMC}\left(\mathrm{kg} / \mathrm{m}^{2}\right)$ & $24.7 \pm 3.2$ & $19.9 / 34.1$ & $25.8 \pm 3.6$ & $19.5 / 31.2$ \\
\hline Cintura $(\mathrm{cm})$ & $80.9 \pm 7.9$ & $68.9 / 107.0$ & $86.6 \pm 9.2$ & $72.0 / 98.0$ \\
\hline RCQ & $0.85 \pm 0.05$ & $0.76 / 0.99$ & $0.87 \pm 0.04$ & $0.80 / 0.91$ \\
\hline Gordura (\%) & $15.7 \pm 6.8$ & $4.3 / 29.2$ & $18.8 \pm 5.8$ & $8.5 / 28.0$ \\
\hline VM 1600m (m.min $\left.{ }^{-1}\right)$ & $200.7 \pm 26.3$ & $125.9 / 246.1$ & - & - \\
\hline $\mathrm{VO}_{2} \max \left(\mathrm{mL} \cdot \mathrm{kg} \cdot \mathrm{min}^{-1}\right)$ & $43.6 \pm 4.6$ & $30.3 / 51.6$ & - & - \\
\hline PAS Repouso (mmHg) & $121 \pm 7$ & $111 / 138$ & $120 \pm 10$ & $107 / 140$ \\
\hline PAS Pico30" (mmHg) & $136 \pm 10^{* *}$ & $120 / 160$ & $136 \pm 13^{* *}$ & $126 / 160$ \\
\hline PAS Pico60" (mmHg) & $138 \pm 10^{* *}$ & $120 / 160$ & $137 \pm 13^{* *}$ & $124 / 162$ \\
\hline PAS Rpós2' (mmHg) & $122 \pm 9$ & $108 / 150$ & $122 \pm 10$ & $112 / 140$ \\
\hline PAD Repouso (mmHg) & $80 \pm 6$ & $70 / 98$ & $80 \pm 8$ & $73 / 98$ \\
\hline PAD Pico30" (mmHg) & $94 \pm 9 * *$ & $80 / 118$ & $93 \pm 10^{* *}$ & $82 / 116$ \\
\hline PAD Pico60" (mmHg) & $97 \pm 9 * *$ & $86 / 124$ & $95 \pm 10^{* *}$ & $84 / 114$ \\
\hline PAD Rpós2' (mmHg) & $82 \pm 10$ & $68 / 116$ & $82 \pm 10$ & $70 / 100$ \\
\hline
\end{tabular}

Nota: IMC: Índice de massa corporal; RCQ: Relação cintura quadril; VM: Velocidade Média; $\mathrm{VO}_{2}$ max: Consumo máximo de oxigénio; PAS: Pressão arterial sistólica; PAD: Pressão arterial diastólica. ${ }^{* *} p<0.01$ quando comparado ao repouso

Tabela 2

Valores de correlação (r) e Effect Size (dentro de parênteses) entre pressão arterial de repouso e reatividade vascular de pressão arterial com indicadores antropométricos $(n=40)$ e aptidão aeróbia $(n=30)$

\begin{tabular}{lccccc}
\hline & $\begin{array}{c}\mathrm{IMC} \\
\left(\mathrm{kg} / \mathrm{m}^{2}\right)\end{array}$ & $\begin{array}{c}\mathrm{CINT} \\
(\mathrm{cm})\end{array}$ & RCQ & Gordura (\%) & $\begin{array}{c}\mathrm{VO}_{2} \mathrm{max} \\
\left(\mathrm{mL}^{\mathrm{k} g} \cdot \mathrm{min}^{-1}\right)\end{array}$ \\
\hline PAS Repouso (mmHg) & $0.36^{*}(0.3)$ & $0.31^{*}(0.3)$ & $0.20(0.2)$ & $0.26(0.2)$ & $-0.07(0.0)$ \\
PAS Pico30" (mmHg) & $0.26(0.2)$ & $0.30^{*}(0.3)$ & $0.22(0.2)$ & $0.18(0.1)$ & $0.03(0.0)$ \\
PAS Pico60" (mmHg) & $0.31^{*}(0.3)$ & $0.35^{*}(0.3)$ & $0.35(0.3)$ & $0.26(0.2)$ & $0.01(0.0)$ \\
PAS Rpós2' (mmHg) & $0.47^{* *}(0.5)$ & $0.42^{* *}(0.4)$ & $0.27(0.2)$ & $0.22(0.2)$ & $-0.01(0.0)$ \\
PAD Repouso (mmHg) & $0.51^{* *}(0.5)$ & $0.46^{* *}(0.5)$ & $0.30^{*}(0.3)$ & $0.38^{* *}(0.4)$ & $-0.27(0.2)$ \\
PAD Pico30" (mmHg) & $0.40^{* *}(0.4)$ & $0.43^{* *}(0.4)$ & $0.26(0.2)$ & $0.29(0.3)$ & $-0.07(0.0)$ \\
PAD Pico60" (mmHg) & $0.41^{* *}(0.4)$ & $0.43^{* *}(0.4)$ & $0.25(0.2)$ & $0.31^{*}(0.3)$ & $-0.13(0.1)$ \\
PAD Rpós2' (mmHg) & $0.54^{* *}(0.6)$ & $0.49^{* *}(0.5)$ & $0.36^{*}(0.3)$ & $0.35^{*}(0.3)$ & $-0.17(0.1)$ \\
\hline
\end{tabular}

Nota: IMC: Índice de massa corporal; CINT: Circunferência da cintura; RCQ: Relação cintura quadril; $\mathrm{VO}_{2}$ max: Consumo máximo de oxigénio; PAS: Pressão arterial sistólica; PAD: Pressão arterial diastólica. ${ }^{*} p<0.05 ;{ }^{*} p<0.01$ 
Tabela 3

Resultados de média $( \pm D P)$ da associação entre pressão arterial de repouso $(\mathrm{mmHg})$ e reatividade vascular de pressão arterial $(\mathrm{mmHg})$ com a aptidão aeróbia

$$
\mathrm{VO}_{2} \max \left(\mathrm{ml} \cdot \mathrm{kg} \cdot \mathrm{min}^{-1}\right)
$$

\begin{tabular}{lccc} 
& $\begin{array}{c}\text { Baixo: } 39.8 \pm 3.6 \\
(\mathrm{n}=14)\end{array}$ & $\begin{array}{c}\text { Alto: } 46.9 \pm 2.4 \\
(\mathrm{n}=16)\end{array}$ & $p$ (Effect Size) \\
\hline PAS Repouso & $121 \pm 7$ & $119 \pm 6$ & $0.48(0.2)$ \\
PAS Pico30" & $136 \pm 10$ & $136 \pm 10$ & $0.91(0.0)$ \\
PAS Pico60" & $138 \pm 10$ & $137 \pm 9$ & $0.72(0.1)$ \\
PAS Rpós2' & $122 \pm 12$ & $122 \pm 6$ & $0.91(0.0)$ \\
PAD Repouso & $82 \pm 7$ & $78 \pm 6$ & $0.12(0.5)$ \\
PAD Pico30" & $96 \pm 10$ & $93 \pm 8$ & $0.40(0.3)$ \\
PAD Pico60" & $99 \pm 11$ & $94 \pm 6$ & $0.19(0.4)$ \\
PAD Rpós2' & $84 \pm 13$ & $81 \pm 5$ & $0.42(0.2)$ \\
\hline
\end{tabular}

Nota: $\mathrm{VO}_{2}$ max: Consumo máximo de oxigénio; PAS: Pressão arterial sistólica; PAD: Pressão arterial diastólica; Rpós2': Recuperação 2 min após CPT

Tabela 4

Resultados de média ( \pm DP) da associação entre indicadores antropométricos com a aptidão aeróbia

\begin{tabular}{lccc}
\hline & \multicolumn{2}{c}{$\mathrm{VO}_{2} \mathrm{max}\left(\mathrm{ml} \cdot \mathrm{kg} \cdot \mathrm{min}^{-1}\right)$} & \\
& $\begin{array}{c}\text { Baixo: } 39.8 \pm 3.6 \\
(\mathrm{n}=14)\end{array}$ & $\begin{array}{c}\text { Alto: } 46.9 \pm 2.4 \\
(\mathrm{n}=16)\end{array}$ & $p$ (Effect Size) \\
\hline Idade $(\mathrm{anos})$ & $25.6 \pm 4.2$ & $25.8 \pm 6.6$ & $0.96(0.0)$ \\
IMC $\left(\mathrm{kg} / \mathrm{m}^{2}\right)$ & $26.3 \pm 3.5$ & $23.4 \pm 2.2$ & $0.01(1.0)$ \\
Cintura $(\mathrm{cm})$ & $84.2 \pm 9.4$ & $78.0 \pm 5.0$ & $0.03(0.8)$ \\
RCQ & $0.8 \pm 0.1$ & $0.8 \pm 0.1$ & $0.89(0.1)$ \\
Gordura $(\%)$ & $19.6 \pm 6.2$ & $12.3 \pm 5.3$ & $0.01(1.0)$ \\
\hline
\end{tabular}

Nota: $\mathrm{VO}_{2}$ max: Consumo máximo de oxigénio; IMC: Índice de massa corporal; RCQ: Relação cintura quadril

Tabela 5

Regressão múltipla entre reatividade vascular de pressão arterial sistólica (PAS) e diastólica (PAD) com os indicadores antropométricos $(n=40)$

\begin{tabular}{llcc}
\hline & Beta & $R^{2}$ Ajustado & $p$ (Effect Size) \\
\hline PAS Pico30" vs. Cintura & 0.30 & 0.09 & $0.05(0.6)$ \\
PAS Pico60" vs. Cintura & 0.35 & 0.12 & $0.02(0.8)$ \\
PAS Rpós2' vs. IMC & 0.81 & 0.22 & $0.01(2.8)$ \\
PAD Pico30" vs. Cintura & 0.43 & 0.18 & $0.01(0.1)$ \\
PAD Pico60" vs. Cintura & 0.43 & 0.19 & $0.01(0.1)$ \\
PAD Rpós2' vs. IMC & 0.54 & 0.29 & $0.01(1.3)$ \\
\hline
\end{tabular}

Nota: IMC: Índice de massa corporal; RCQ: Relação cintura quadril; Rpós2': Recuperação 2 min após CPT 
possível identificar que apenas a CC se mostrou preditora da $\mathrm{RV}_{\mathrm{PA}}$ durante o teste de estresse cardiovascular, com o IMC tendo importância na predição da $\mathrm{RV}_{\mathrm{PA}}$ na recuperação do estresse induzido (Tabela 5). Por outro lado, a aptidão aeróbia não apresentou associação direta com a $\mathrm{RV}_{\mathrm{PA}}$ de homens adultos (Tabelas 2 e 3 ).

Diversos indicadores de adiposidade corporal têm sido utilizados na área da saúde e a CC e o IMC têm sido destacados na predição da HAS (Tuan, Adair, Stevens \& Popkin, 2010). Carneiro et al. (2003) ao analisarem indivíduos com sobrepeso e obesidade, demonstraram um aumento significativo na prevalência de HAS, especialmente nos participantes com IMC $\geq 30 \mathrm{~kg} / \mathrm{m}^{2}$, sugerindo que o incremento dos depósitos de gordura corporal aumentam o risco de HAS. Esses resultados corroboram os achados do presente estudo, os quais apresentam correlação positiva da PA de repouso, e em adicional da $R V_{\mathrm{PA}}$, com indicadores antropométricos, principalmente com a CC (gordura abdominal) e IMC de homens adultos (Tabela 2).

$\mathrm{O}$ mecanismo que explica a associação da adiposidade corporal com a $\mathrm{RV}_{\mathrm{PA}}$ pode ser parcialmente explicado. $\mathrm{O}$ aumento da adiposidade corporal está relacionado com uma maior atividade nervosa simpática (Kuniyoshi et al., 2003; Ribeiro et al., 2001) e com uma possível ativação do sistema reninaangiotensina, o qual estaria diminuindo a biodisponibilidade de óxido nítrico e por conseguinte aumentando a rigidez arterial e a PA (Christou et al., 2005). No presente estudo, a CC e o IMC se correlacionaram com a $\mathrm{PA}$ de repouso e com a $\mathrm{RV}_{\mathrm{PA}}$, essa última induzida pelo Cold Pressor Test (Tabela 2), o qual atua via um possível reflexo neurogénico e aumento do tónus simpático (Wood et al., 1984). Kuniyoushi et al. (2003) avaliaram a PA e a ativação simpática em mulheres obesas e magras. Estes autores também utilizaram o Cold Pressor Test e concluíram que a obesidade aumenta o tónus simpático e a $\mathrm{RV}_{\mathrm{PA}}$ durante o estresse induzido.
Apesar da falta de correlação entre aptidão aeróbia e $\mathrm{RV}_{\mathrm{PA}}$ (Tabela 2), optou-se no presente estudo por investigar uma possível associação entre essas variáveis por meio de diferentes estratos de $\mathrm{VO}_{2} \max$ (Tabela 3 ). Sendo assim, foi possível verificar que nenhuma das variáveis da $R V_{P A}$ para PAS e PAD (Pico30", Pico60" e Rpós2') diferiram entre os grupos com $\mathrm{VO}_{2}$ max abaixo e acima da média constatada no presente estudo ( $p>$ 0.05). Nessa mesma direção, ao comparar a $\mathrm{RV}_{\mathrm{PA}}$ entre grupos com $\mathrm{VO}_{2} \max$ abaixo do percentil 10 e acima do percentil 90 do presente estudo, mais uma vez não foram identificadas diferenças significativas $(p>0.05$; resultados não apresentados). Esses achados evidenciam que $\mathrm{o}$ efeito independente $\mathrm{e}$ cardioprotetor do $\mathrm{VO}_{2} \max$ na $\mathrm{RV}_{\mathrm{PA}}$ de homens adultos não é significativo, corroborando aos achados de Christou et al. (2005) os quais destacam que o $\mathrm{VO}_{2}$ max, quando analisado em relação a variáveis antropométricas, não apresenta correlações independentes com fatores de risco hemodinâmicos para a doença cardiovascular.

Entretanto, ao se verificar a associação entre $\mathrm{VO}_{2}$ max abaixo e acima da média com indicadores antropométricos, constatou-se que a CC, o IMC e o \%Gordura apresentaram-se reduzidos no grupo com maior $\mathrm{VO}_{2} \mathrm{max}$ (Tabela 4; $p<0.05$ ). Nesse sentido, apesar da falta de uma associação direta entre aptidão aeróbia e $\mathrm{RV}_{\mathrm{PA}}$, O $\mathrm{VO}_{2} \max$ apresentou associação indireta, via indicadores antropométricos, com a $\mathrm{RV}_{\mathrm{PA}}$, uma vez que indivíduos com maiores valores de $\mathrm{VO}_{2}$ max apresentaram menores índices de adiposidade corporal (CC e IMC), os quais influenciam direta e independentemente na $\mathrm{RV}_{\mathrm{PA}}$ de homens adultos (Tabela 2).

As correlações independentes, porém, moderadas entre indicadores antropométricos e $\mathrm{RV}_{\mathrm{PA}}$ (Tabela 2), bem como, a predição significativa da CC e do IMC na $\mathrm{RV}_{\mathrm{PA}}$ (Tabela 5), sugerem a investigação de outras variáveis, não relacionadas ao fenótipo, na explicação da $\mathrm{RV}_{\mathrm{PA}}$ de homens adultos. Dessa forma, traba- 
lhos investigando genes candidatos na variabilidade das respostas de $R_{P A}$ são necessários. Esses estudos podem contribuir com o conhecimento relacionado aos componentes genéticos e ambientais que interferem em fatores de risco cardiovasculares, pois, com o prévio conhecimento dessas variáveis, estratégias atenuando o risco futuro podem ser encorajadas na população.

\section{CONCLUSÕES}

Conclui-se que indicadores antropométricos, mas não a aptidão aeróbia, se associam direta e independentemente com a $\mathrm{RV}_{\mathrm{PA}}$ de homens adultos. Ainda, foi possível identificar que a $\mathrm{CC}$ se mostrou preditora da $\mathrm{RV}_{\mathrm{PA}}$ durante o estresse cardiovascular induzido, com o IMC tendo importância na predição da recuperação da $\mathrm{RV}_{\mathrm{PA}}$ de homens adultos. Apesar da falta de uma associação direta entre aptidão aeróbia e $\mathrm{RV}_{\mathrm{PA}}$, o $\mathrm{VO}_{2}$ max apresentou associação indireta, via indicadores antropométricos, com a $\mathrm{RV}_{\mathrm{PA}}$ de homens adultos.

Agradecimentos:

Ao CNPq pelo apoio financeiro com bolsa AT-NM.

Conflito de Interesses:

Nada a declarar.

Financiamento:

CNPq (proc. 503598/2010).

\section{REFERÊNCIAS}

Almeida, J. A., Pardono, E., Sotero, C. R., Magalhães, G., Simões, H. G., \& Campbell, C. S. G. (2010). Validação de equações de predição em estimar o VO2máx de brasileiros jovens a partir do desempenho em corrida de $1.600 \mathrm{~m}$. Revista Brasileira de Medicina do Esporte, 16(1), 57 60.

Bland, M. J., \& Altman, G. D. (1986). Statistical methods for assessing agreement between two methods of clinical measurement. Lancet, 1 (8476), 307-310.

Bring, J. F., \& Oerting, H. (1933). Vasomotor response of normal and hypertensive individuals to standart stimulus (cold). Minnesota Medicine, 16, 481-486.

Brownley, K. A., Hinderliter, A. L., West, S. G., Girdler, S.S., Sherwood, A., \& Light, K. C. (2003). Sympathoadrenergic mechanisms in reduced hemodynamic stress responses after exercise. Medicine \& Science in Sports \& Exercise, 35(6), 978-986.

Callaway, C. W., Chumlea, W. C., Bouchard, C. R., Himes, J. H., Lohman, T. G., \& Martim, A. D. (1988). Circumferences. In T. G. Lohman, A. F. Roche, \& R. Martorell (Eds.), Anthropometric standardization reference manual (pp. 39-54). Champaign, Il: Human Kinetics.

Carneiro, G., Faria, A., Ribeiro, F., Guimarães, A., Lerário, D., \& Ferreira, S. (2003). Influência da distribuição da gordura corporal sobre a prevalência de hipertensão arterial e outros fatores de risco cardiovascular em indivíduos obesos. Revista da Associação Medica Brasileira, 38, 122127. doi: 10.1590/S0104-42302003000300036

Chobanian, A. V., Bakris, G. L., Black, H. R., Cushman, W. C., Green, L. A., Izzo, Jr., Jones, W. D., ... White, Jr. (2003). The Seventh Report of the Joint National Committee on Prevention, Detection, Evaluation, and Treatment of High Blood Pressure: The JNC 7 Report. The Journal of the American Medical Association, 289, 25602571.

Christou, D., Gentile, C., Desouza, C., Seals, D., \& Gates, P. (2005). Fatness is a better predictor for cardiovascular disease risk factor profile than aerobic fitness in healthy men. Circulation, 19, 122-127. doi: 10.1161/01.CIR.0000161818. 28974.1

Flaa, A., Mundal, H., Eide, I., Kjelsen, S., \& Rostrup, M. (2006). Sympathetic activity and cardiovascular risk factors in young men in the low, normal, and high blood pressure rangers. Hypertenson, 47, 396-402. doi: 10.1161/01.HYP. 0000203952.27988.79

Gaesser, A. Angadi, S., \& Sawyer, J. (2011). Exercise and diet, independent of weight loss, improve cardiometabolic risk profile in overweight and obese individuals. The Physician Sports Medicine, 39, 87-97. doi: 10.3810/psm.2011.05.1898

Galetta, F., Franzoni, F., Plantinga, Y., Ghiadoni, L., Rossi, M., Prattichizzo, F., ... Taddei, G. 
(2006). Ambulatory blood pressure monitoring and endothelium-dependent vasodilation in the elderly athletes. Biomedicine \& Pharmacotherapy, 60(8), 443-447.

Guedes, D. P., \& Guedes, J. E. (1997). Exercício físico na promoção da saúde. São Paulo: Midiograf.

Hair, J. F., Black, W. C., Babin, B. J., Anderson, R. E., \& Tatham, R. L. (2009). Análise multivariada de dados ( $6^{\mathrm{a}}$ ed). Porto Alegre: Bookman.

Hines, E. \& Brown, G. (1936). The cold test pressor for measuring the reactibility of the blood pressure: Data concerning 571 normal and hypertensive subjects. The American Heart Journal, 11, 1-9.

Hoehner, C. M., Soares, J., Parra, P. D., Ribeiro, I.C., Joshu, C. E., \& Pratt, M. (2008). Physical activity interventions in Latin America: A systematic review. American Journal of Preventive Medicine, 34(3), 224-233. doi: 10.1016/j.amepre.2007. 11.016

Jackson, A. S., \& Pollock, M. L. (1978). Generalized equations for predicting body density of men. British Journal of Nutrition, 40(3), 497-504.

Kuniyoshi, F., Trombetta, I., Batalha, L., Rondon, M., Laterza, M., \& Gowdak, M. (2003). Abnormal neurovascular control during sympathoexcitation in obesity. Obesity Research, 11, 1411-1419.

Palou, A., Serra, F., Bonet, M., \& Picó, C. (2000). Obesity: Molecular bases of a multifactorial problem. European Journal of Nutrition, 39, 127144.

Perloff, D., Grim, C., Flack, J., Frohlick, E., Hill, M., Mcdonald, M., \& Morgenstern, B. Z. (1993). Human blood pressure determination by sphygmomanometry. Circulation, 88(5), 24602470. doi: 10.1161/01.CIR.88.5.2460

Ribeiro, M. M., Trombetta, I. C., Batalha, L. T., Rondon, M. U., Forjaz, C. L., \& Barreto, A. C. (2001). Muscle sympathetic nerve activity and hemodinamic alterations in middle-aged obese women. Brazilian Journal of Medical and Biological Research, 34, 475-478. doi: 10.1590/S0100879X2001000400006

Siri, W. E. (1961). Body composition from fluids space and density: Analysis of methods. In J. Brozek \& A. Henschel (Eds.), Techniques for measuring body composition (pp. 223-224). Wash- ington, DC: National Academy of Sciences, National Research Council.

Stergiou, G. S., Tzamouranis, D., Protogerou, A., Nasothimiou, E., \& Kapralos, C. (2008). Validation of the Microlife Watch BP Office professional device for office blood pressure measurement according to the International protocol. Blood Press Monitoring, 13(5), 299-303. doi: 10.1097/MBP.0b013e3283057af6

Su, T. C., Lee, Y. T., Chou, S., Hwang, W. T., Chen, C. F., \& Wang, J. D. (2006). Twenty-four-hour ambulatory blood pressure and duration of hypertension as major determinants for intimamedia thickness and atherosclerosis of carotid arteries. Atherosclerosis, 184(1), 151-156. doi: 10.1016/j.atherosclerosis.2005.03.041

Thompson, P. D., Buchner, D., Pina, I. L., Balady, G. J., Williams, M. A., Marcus, B. H., Berra, K., ... Wenger, N. K. (2003). Exercise and physical activity in the prevention and treatment of atherosclerotic cardiovascular disease. Circulation, 107, 3109-3116. doi: 10.1161/01.CIR.0000075 572.40158. 77

Triches, R. M., \& Giugliani, E. (2005). Obesidade, práticas alimentares e conhecimentos de nutrição em escolares. Revista de Saúde Pública, 39(6), 541-547. doi: 10.1590/S0034-89102005 000400004

Tuan, N. T., Adair, L. S., Stevens, J., \& Popkin, B. M. (2010). Prediction of hypertension by different anthropometric indices in adults: The change in estimate approach. Public Health Nutrition, 13(5), 639-646. doi: 10.1017/S136898000999 1479

Vincent, J. (2011). The paradox of obesity and cardiovascular disease risk: Time to change labels. Clinical Pharmacology Therapeutics, 90(1), 3-9. doi: 10.1038/clpt.2011.121

Wilson, P. W., D’Agostino, R. B., Sullivan, L., Parise, H., \& Kannel, W. B. (2002). Overweight and obesity as determinants of cardiovascular risk: The Framingham experience. Archives of International Medicine, 162(16), 1867-1872. doi: 10.1001/archinte.162.16.1867

Wood, D. L., Sheps, S. G., Elveback, L. R., Schirger, A. (1984). Cold pressor test as a predictor of hypertension. Hypertension, 6(3), 301-306. doi: 10.1161/01.HYP.6.3.301 